\title{
Penetrance of symbiont-mediated parthenogenesis is driven by reproductive rate in a parasitoid wasp
}

\author{
Amelia R.I. Lindsey ${ }^{1}$ ， Richard Stouthamer ${ }^{\text {Corresp. } 1}$ \\ ${ }^{1}$ Department of Entomology, University of California, Riverside, Riverside, California, United States \\ Corresponding Author: Richard Stouthamer \\ Email address: richard.stouthamer@ucr.edu
}

Trichogramma wasps are tiny parasitoids of lepidopteran eggs, used extensively for biological control. They are often infected with the bacterial symbiont Wolbachia, which converts Trichogramma to an asexual mode of reproduction, whereby females develop from unfertilized eggs. However, this Wolbachia-induced parthenogenesis is not always complete, and previous studies have noted that infected females will produce occasional males in the lab. The conditions that reduce penetrance of the parthenogenesis phenotype are not well understood. We hypothesized that more ecologically relevant conditions of limited host access will sustain female-biased sex ratios. After restricting access to host eggs, we found a strong relationship between reproductive rate and sex ratio. By limiting reproduction to one hour a day, wasps could sustain up to $100 \%$ effective parthenogenesis for one week, with no significant impact on total fecundity. Reproductive output in the first 24-hours appears to be critical to the total sex ratio of the entire brood. Limiting oviposition in that period resulted in more effective parthenogenesis after one week, again without any significant impact on total fecundity. Our data suggest that this phenomenon may be due to the depletion of Wolbachia when oviposition occurs continuously, whereas Wolbachia titers may recover when offspring production is limited. In addition to the potential to improve mass rearing of Trichogramma for biological control, findings from this study help elucidate the context-dependent nature of a pervasive symbiotic relationship. 
1 Penetrance of symbiont-mediated parthenogenesis is driven by reproductive rate in a

2

parasitoid wasp

3

$4 \quad$ Amelia R. I. Lindsey ${ }^{1}$ and Richard Stouthamer ${ }^{*}$

5

$6{ }^{1}$ Department of Entomology, University of California Riverside, Riverside, California, 92521,

7 USA

8

$9 *$ Corresponding Author:

10 Richard Stouthamer

11 Email: richard.stouthamer@ucr.edu 


\section{Abstract}

13 Trichogramma wasps are tiny parasitoids of lepidopteran eggs, used extensively for biological

14 control. They are often infected with the bacterial symbiont Wolbachia, which converts

15 Trichogramma to an asexual mode of reproduction, whereby females develop from unfertilized

16 eggs. However, this Wolbachia-induced parthenogenesis is not always complete, and previous

17 studies have noted that infected females will produce occasional males in the lab. The conditions

18 that reduce penetrance of the parthenogenesis phenotype are not well understood. We

19 hypothesized that more ecologically relevant conditions of limited host access will sustain

20 female-biased sex ratios. After restricting access to host eggs, we found a strong relationship

21 between reproductive rate and sex ratio. By limiting reproduction to one hour a day, wasps could

22 sustain up to $100 \%$ effective parthenogenesis for one week, with no significant impact on total

23 fecundity. Reproductive output in the first 24-hours appears to be critical to the total sex ratio of

24 the entire brood. Limiting oviposition in that period resulted in more effective parthenogenesis

25 after one week, again without any significant impact on total fecundity. Our data suggest that this

26 phenomenon may be due to the depletion of Wolbachia when oviposition occurs continuously,

27 whereas Wolbachia titers may recover when offspring production is limited. In addition to the

28 potential to improve mass rearing of Trichogramma for biological control, findings from this

29 study help elucidate the context-dependent nature of a pervasive symbiotic relationship.

\section{Key Words}

32 Wolbachia, Trichogramma, sex ratio, asexual, reproductive modification, symbiosis 


\section{Introduction}

Wolbachia is a maternally transmitted, symbiotic bacterium that inhabits numerous arthropods and nematodes. Its ubiquity can be attributed to both fitness advantages for the host, and reproductive modifications of the host. Known reproductive modifications include cytoplasmic incompatibility, male-killing, feminization, and parthenogenesis-induction (Werren et al. 2008), all of which increase the relative fitness of infected females, thus allowing Wolbachia to spread through a population (Hoffmann et al. 2011; Turelli \& Hoffmann 1991). Parthenogenesisinducing Wolbachia infect haplodiploid species and result in the production of females without the need for a mate. This is accomplished through converting unfertilized eggs (that would otherwise develop as males) to diploid eggs, which then develop as females (Gottlieb et al. 2002; Pannebakker et al. 2004; Stouthamer \& Kazmer 1994).

There is a large body of research indicating that the phenotypes Wolbachia induces are context dependent, with a range of genetic and environmental factors influencing the penetrance of the manipulation (proportion of individuals displaying the phenotype)(Mouton et al. 2007; Pascal et al. 2004; Serbus et al. 2015; Wiwatanaratanabutr \& Grandjean 2016). These are important considerations for several reasons. Firstly, with symbionts under exploration for the control of target pest species (Bourtzis et al. 2014; Hoffmann et al. 2011; Hoffmann et al. 2015; Walker et

54 al. 2011), it is critical that we understand the dynamics that result in the desired host-symbiont 55 extended phenotype, and the persistence of the infection in the target population. Secondly, the persistence of a symbiont in a host population, and expression of resulting phenotypes will affect 
58 the reproductive modification or manipulation, relative fitness costs or benefits for the host, and

59 the proportion of infected individuals in the population all play into the ability of Wolbachia to

60 spread and maintain itself in a population (Hoffmann et al. 2011; Hoffmann et al. 1990; Turelli

61 \& Hoffmann 1995).

62

63 Changes in host genotype or the introduction to a novel host can result in altered Wolbachia

64 titers (Mouton et al. 2007; Watanabe et al. 2013), failure to induce the anticipated phenotype

65 (Bordenstein et al. 2003; Grenier et al. 1998; Huigens et al. 2004; McGraw et al. 2001; Reynolds

66 et al. 2003), reduced maternal transmission, and the eventual loss of the symbiont from a

67 population (Huigens et al. 2004; Newton et al. 2015). Additionally, there are well-characterized

68 relationships between several environmental factors and the penetrance of Wolbachia-mediated

69 phenotypes. High temperatures will reduce Wolbachia titers and result in poor host manipulation

70 (Bordenstein \& Bordenstein 2011; Hurst et al. 2000; Pascal et al. 2004). The same result has

71 been found for antibiotic treatments: the higher the antibiotic dose, the lower the symbiont titer,

72 and the lower the penetrance of the reproductive manipulation (Zchori-Fein et al. 2000). In the

73 case of cytoplasmic incompatibility-inducing Wolbachia, this means heat-treated male offspring

74 of are incapable of inducing cytoplasmic incompatibility, or only do so weakly (Clancy \&

75 Hoffmann 1998). In the case of parthenogenesis-inducing Wolbachia, antibiotic treated mothers

76 produce increasingly more sons at lower Wolbachia titers (Stouthamer \& Mak 2002; Zchori-Fein

77 et al. 2000). Many of these studies point to a "threshold" level of infection that is critical for

78 host-manipulation (Bordenstein \& Bordenstein 2011; Hurst et al. 2000; Ma et al. 2015), and a

79 positive correlation between Wolbachia titers and expression of the manipulation (Bourtzis et al.

80 1996; Breeuwer \& Werren 1993; Ikeda et al. 2003; Pascal et al. 2004; Zchori-Fein et al. 2000). 
81

82 The effect of removing or reducing Wolbachia titers is well documented for wasps in the genus

83 Trichogramma (Pintureau et al. 1999; Stouthamer et al. 1990a; Tulgetske \& Stouthamer 2012).

84 Trichogramma are minute parasitoid wasps in the superfamily Chalcidoidea, frequently infected 85 with parthenogenesis-inducing Wolbachia (Stouthamer et al. 1993; Stouthamer et al. 1990a;

86 Stouthamer et al. 1990b). Like other hymenopterans, Trichogramma are haplodiploid:

87 unfertilized eggs typically develop into males, and fertilized eggs into females (Stouthamer et al.

88 1990a). In Trichogramma, parthenogenesis-inducing Wolbachia restores diploidy of unfertilized

89 eggs through via a failed anaphase in which chromosomes do not separate during the egg's first

90 mitotic division (Stouthamer \& Kazmer 1994). For Trichogramma, increased doses of heat

91 reduce bacterial titers and lead to the production of increasingly more males and sexually

92 aberrant individuals (Pascal et al. 2004; Stouthamer 1997; Tulgetske \& Stouthamer 2012). It is

93 not clear however, why males are occasionally produced in the absence of antibiotics or

94 increased temperature regimes (Hohmann et al. 2001; Stouthamer \& Luck 1993).

95

96 The production of males could be useful in determining what factors control the expression of

97 the symbiont phenotype. Preliminary studies that suggest that limited access to host eggs results

98 in more female-biased sex ratios (Hohmann et al. 2001; Legner 1985; Stouthamer \& Luck 1993).

99 However, the relationship between access to host eggs and progeny sex ratio has not been teased

100 apart. Prior to the discovery of Wolbachia as a parthenogenesis-inducer, fecundity patterns were

101 suggested to affect the resulting sex ratio in the parasitoid wasp Muscidifurax uniraptor (Legner

102 1985). It was later discovered that Muscidifurax uniraptor is a host for parthenogenesis-inducing

103 Wolbachia, and that Wolbachia titers are positively correlated with the proportion of females 
104 produced (Zchori-Fein et al. 2000). Here, we use a line of Trichogramma pretiosum fixed for

105 Wolbachia infection to explore the relationship between patterns of offspring production and sex 106 ratios.

107

108

2. Materials and Methods

109

(a) Trichogramma Colonies

110 Isofemale lines of Trichogramma pretiosum are maintained in $12 \times 75 \mathrm{~mm}$ glass culture tubes

111 stopped with cotton and incubated at $24^{\circ} \mathrm{C}, \mathrm{L}: \mathrm{D}=16: 8$. Every 11 days colonies are given honey

112 and egg cards made of irradiated Ephestia kuehniella host eggs (Beneficial Insectary, Guelph,

113 Ontario, Canada) afixed to card stock with double-sided tape. Species identification was

114 confirmed by molecular protocols from Stouthamer et al. (1999). We used the "Insectary" line,

115 collected from the Puira Valley of Peru, which has been maintained in a commercial insectary

116 since 1966 (Beneficial Insectary, Guelph, Ontario, Canada). The Insectary line exhibits

117 thelytokous reproduction: females hatch from unfertilized eggs, indicating infection with

118 Wolbachia. Infection status was confirmed by PCR following Werren and Windsor (2000).

119

120 (b) Host Access Experiments

121 Individual Insectary line wasps from a single generation were isolated during the pupal stage to 122 ensure virginity. Darkened Ephestia eggs (indicating a developing Trichogramma pupa) were

123 removed from cards using a paintbrush and water, and isolated in $12 \times 75 \mathrm{~mm}$ glass culture tubes

124 stopped with cotton. Only wasps that hatched singly from an Ephestia egg on day one were used

125 in trials, ensuring size-matched, age-matched, virgin wasps. Upon emergence, wasps were

126 subjected to one of four treatments to determine how access to host eggs, and resultant offspring 
127 production, affects Wolbachia titers and sex ratio (here defined as percentage females among all

128 offspring). Twenty replicate individual wasps were used for each of the following treatments: 1)

129 a surplus of fresh host eggs every 24 hours for seven days, 2) a surplus of fresh host eggs for 24

130 hours every other day, for seven days, 3) a surplus of fresh host eggs for only one hour a day, for

131 seven days, or 4) immediate collection into $100 \%$ ethanol upon adult emergence (Figure 1A).

132 Treatments two and three were designed to restrict access to host eggs, and treatment four was

133 designed to collect data on Wolbachia titers in newly emerged wasps. For treatment three,

134 exposure to the fresh egg card was performed at the same time each day, from 10:45AM -

135 11:45AM. Egg cards were isolated in individual tubes after the exposure period, ensuring no

136 further parasitization. All mothers, regardless of treatment, were provided with a streak of fresh

137 honey every 24 hours. On day eight, all mothers from the first three treatments were collected

138 into $100 \%$ ethanol. All offspring from each isolated egg card were allowed to develop, and

139 collected into $100 \%$ ethanol within 24 hours of adult emergence. Offspring were counted and

140 identified as female, male, or intersex based on antennal morphology. Wolbachia quantification

141 (see below) was performed on mothers and select progeny.

142

143 (c) Quantification of Wolbachia Titers

144 Total DNA was extracted from wasps using a Chelex method (Walsh et al. 1991) as

145 implemented by Stouthamer et al (Stouthamer et al. 1999). Gene sequences from the single-copy

146 Trichogramma pretiosum gene wingless, and the Wolbachia 16S gene were identified from the

147 genome assemblies (GenBank Accession Numbers: JARR00000000 and LKEQ01000000,

148 (Lindsey et al. 2016)). Trichogramma pretiosum wingless was identified through BLAST

149 searches of the genome, using the Trichogramma evanescens homolog as a query (GenBank 
150 Accession Number: GQ368153.1). Specific primers (Table 1) were designed to amplify variable

151 regions of these two genes, using primer3 (Untergasser et al. 2012). Primer specificity was

152 checked computationally with Primer-BLAST (Ye et al. 2012), and against extractions of the

153 moth host eggs, E. kuehniella, which has an orthologous copy of wingless, and is infected with

154 its own strain of Wolbachia. qPCR was performed in $20 \mu$ reactions containing $1 \mathrm{x}$ ThermoPol ${ }^{\mathrm{TM}}$

155 buffer (New England Biolabs), $0.4 \mu \mathrm{M}$ each primer, 200nM each of dATP, dCTP, and dGTP,

$156400 \mathrm{nM}$ dUTP, $1 \mathrm{mM} \mathrm{MgCl} 2,0.5 \mathrm{x}$ EvaGreen ${ }^{\circledR}$ (Biotium), $1 \mathrm{U}$ Taq polymerase (New England

157 Biolabs), and $2 \mu 1$ of sample. Reactions were denatured at $95^{\circ} \mathrm{C}$ for 3 minutes, followed by 35

158 cycles of $95^{\circ} \mathrm{C}$ for 20 seconds, $58^{\circ} \mathrm{C}$ for 20 seconds, and $72{ }^{\circ} \mathrm{C}$ for 20 seconds. All samples

159 were run in triplicate alongside calibration standards and negative controls on a Rotor-Gene ${ }^{\circledR} \mathrm{Q}$

160 (QIAGEN). Relative Wolbachia titers were determined with the $\Delta \Delta \mathrm{Ct}$ method (Livak \&

161 Schmittgen 2001) with normalization to wingless. When testing titers in offspring, we did not

162 correct wingless quantification for ploidy levels between males and females as there is evidence

163 that most of the somatic tissues in males are diploid (Aron et al. 2005).

164

165 (d) Limiting Host Access in the First 24 Hours

166 Given the results of the initial host access treatments (Figure 1A), we set up a second trial to

167 determine the impact of oviposition in the first 24-hour period. Wasps were isolated from a

168 single generation of the Insectary line, and were age and size matched, as before. 12 replicate

169 individual wasps were subjected to either of the following treatments: 1) constant access to fresh

170 host eggs every 24 hours (same as treatment 1 in the first experiments), or, 2) one-hour access to

171 an egg card on day one (10:45 - 11:45AM), followed by constant access to fresh egg cards every

17224 hours starting day two (Figure 1B). Trials were carried out for seven days. Again, mothers 
173 received fresh honey every 24 hours, and egg cards were isolated after the exposure period.

174 Offspring were allowed to emerge, then counted and identified as female, male, or intersex.

175

176 (e) Statistics

177 Statistical analyses and data visualization were performed in $\mathrm{R}$ version 3.1.2. We used a

178 generalized linear mixed-effects model (GLMM) to assess variation in sex ratios (proportions

179 female and non-female offspring) between treatments using treatment and day of the trial as

180 fixed effects, individual wasp identity as a random effect to account for repeated measures, and a

181 binomial error distribution (Bates et al. 2014). We followed up significant effects of treatment

182 with pairwise GLMs, followed by Bonferroni corrections for multiple testing. To assess variation

183 in fecundity among treatments, we used a GLMM with treatment and day of the trial as fixed

184 effects, individual wasp identity as a random effect, and a Poisson error distribution. Here too,

185 we separately assessed variation in total fecundity with a GLM using treatment as a fixed effect,

186 and a Poisson error distribution. We assessed variation in cumulative sex ratio with a GLMM,

187 using cumulative fecundity and treatment as fixed effects, and individual wasp as a random

188 effect. Differences in Wolbachia titers between mothers of different host access treatments were

189 assessed with a one-way ANOVA including treatment as a fixed effect, followed by Tukey

190 Honest Significant Difference for post hoc testing. Differences in Wolbachia titer between

191 offspring were determined with a linear mixed-effects model, with offspring sex as a fixed effect

192 and mother's identity as a random effect (Bates et al. 2014).

193

194 3. Results

195 (a) Sex Ratios are More Female Biased When Host Access is Systematically Restricted 
196 To determine how reproductive rate affects sex ratio, we subjected wasps to treatments with

197 different degrees of accessibility to host eggs for seven days. We found a significant effect of the

198 interaction between treatment and day of trial for sex ratio (Figure $2 \mathrm{~A} ; \chi^{2}=7.38, \mathrm{p}=0.0250$ ).

199 Daily sex ratio significantly differed by treatment (Figure $2 \mathrm{~A} ; \chi^{2}=77.38, \mathrm{p}<0.001$ ) and over

200 time (Figure $2 \mathrm{~A} ; \chi^{2}=326.07, \mathrm{p}<0.001$ ). Wasps in treatment three, where access to host eggs

201 was for only one hour a day, consistently produced the most female-biased sex ratios. Treatment

202 one, where wasps had constant access to host eggs, produced the least female-biased sex ratios,

203 with male-bias increasing over time. Wasps subjected to treatment two (hosts every other day)

204 produced intermediate sex ratios. These patterns resulted in overall brood sex ratios that were

205 significantly different between treatments (Figure $2 \mathrm{~B} ; \chi^{2}=59.72, \mathrm{p}<0.001$ ).

206

207 Similarly, we found a significant effect of the interaction between treatment and day of trial for

208 fecundity (Figure $2 \mathrm{C} ; \chi^{2}=181.81, \mathrm{p}<0.001$ ). Levels of daily fecundity significantly differed by

209 treatment (Figure $2 \mathrm{C} ; \chi^{2}=154.65, \mathrm{p}<0.001$ ), and over time (Figure $2 \mathrm{C} ; \chi^{2}=817.83, \mathrm{p}<$

210 0.001). Treatment one wasps produced very high numbers of offspring on day one, and fecundity

211 for the remainder of the trial decayed exponentially. In contrast, reproduction by treatment three

212 wasps was initially lower, but did not drop exponentially as did the reproduction of treatment one

213 wasps. Treatment two wasps had an intermediate level of reproductive output throughout the

214 trial. Unlike total sex ratios, overall fecundity was not significantly different between treatments

215 (Figure 2D; $\chi^{2}=4.43, \mathrm{p}=0.1091$ ).

216

217 (b) Periodicity of Host Access, Not Cumulative Fecundity Drives Sex Ratios 
218 To show that prior offspring production alone was not the driver of sex ratio, we tracked

219 cumulative fecundity and cumulative sex ratios for the duration of the trial, and found a

220 significant effect of treatment on cumulative sex ratio (Figure $3 ; \chi^{2}=38.795, p<0.001$ ). The

221 most restrictive treatment (three) results in the production of almost all female offspring. At the

222 same point in total reproductive output, wasps in treatments one and two are producing

223 significantly fewer females (Figure 3). This shows that it is the restricted access to hosts, and not

224 the total number of offspring produced up to that point that is maintaining the production of more

225 female offspring in treatments two and three.

226

227 (c) Wasps Have Higher Wolbachia Titers Prior to Oviposition

228 We determined Wolbachia titers in mothers from the first four treatments (Figure 1A), and

229 detected significant differences between treatments (Figure $4 \mathrm{~A} ; \mathrm{F}_{3,70}=5.559, \mathrm{p}=0.002$ ). The

230 wasps from treatment four that were collected immediately upon emergence had the highest

231 average Wolbachia titers, but they were not significantly different from wasps in treatment three

232 (one hour a day access) $(\mathrm{p}=0.280)$. Treatments one and two (constant access, and constant

233 access every other day, respectively) resulted in mothers, on day eight, with significantly lower

234 Wolbachia titers relative to immediately collected wasps $(\mathrm{p}=0.033$, and $\mathrm{p}=0.003$ respectively).

235 However, there was no significant difference between treatments one and two $(\mathrm{p}=0.805)$, even

236 though egg card access was restricted in treatment two.

237

238 (d) Wolbachia Titers are Higher in Wasps That Have Not Recently Oviposited

239 We hypothesized that male offspring would have lower Wolbachia titers, as a consequence of

240 having received an insufficient quantity of Wolbachia from their mother to induce gamete 
241 duplication. We quantified Wolbachia titers of three female offspring and three male offspring,

242 from each of three mothers from treatment one (Figure 1A). After accounting for differences

243 among mothers, Wolbachia titer differed significantly between male and female progeny, with

244 female titers that were more than three times higher than in males (Figure $4 \mathrm{~B} ; \chi^{2}=22.22, \mathrm{p}<$

245 0.001). With the exception of one male, all female offspring had higher titers than their male

246 siblings.

247

248 (e) Reproductive Output in the First 24-Hours Significantly Affects Sex Ratios After One Week

249 Given the finding that the largest difference in fecundity between treatments one and three was

250 during the first 24 hours, we set up a second set of experiments in which one group of wasps'

251 access to egg cards was restricted only on day one, and a second group of wasps had constant

252 access to host eggs (Figure 1B). Under these conditions, we found no interactive effect of

253 treatment and day on sex ratio (Figure $5 \mathrm{~A} ; \chi^{2}=1.90, \mathrm{p}=0.1677$ ). However, we found a

254 significant effect of treatment (Figure $5 \mathrm{~A} ; \chi^{2}=8.75, \mathrm{p}=0.0031$ ), and a significant effect of day

255 (Figure $5 \mathrm{~A} ; \chi^{2}=201.46, \mathrm{p}<0.001$ ) on sex ratio. The experimental treatment (only one hour

256 with an egg card on day one) maintained higher female-biased sex ratios for the duration of the

257 trial. The overall sex ratio of the experimental treatment was significantly more female-biased

258 than the control (Figure 5B; $\chi^{2}=24.40 \mathrm{p}<0.001$ ). In contrast to the sex-ratio results for these

259 trials, we found a significant interactive effect of treatment and day on fecundity (Figure $5 \mathrm{C} ; \chi^{2}$

$260=7.79, \mathrm{p}=0.0053$ ), as well as a significant effect of day alone (Figure $5 \mathrm{C} ; \chi^{2}=395.74, \mathrm{p}<$

261 0.001). Importantly, there was no significant difference in total fecundity between treatments

262 (Figure 5D; $\chi^{2}=1.83, \mathrm{p}=0.1761$ ).

263 


\section{4. Discussion}

265 Based on the established relationship between Wolbachia titers and the parthenogenesis-

266 phenotype (Pascal et al. 2004; Stouthamer 1997; Tulgetske \& Stouthamer 2012; Zchori-Fein et

267 al. 2000), and previous research on Muscidifurax uniraptor that showed sex ratios changed with

268 reproductive patterns (Legner 1985), we hypothesized that reproductive rate might mediate the

269 level of male production in an asexual line of Trichogramma. Restricted access to hosts is likely

270 the more ecologically relevant condition, so the males produced under high host availability

271 conditions in the lab would not be produced under field conditions. In natural settings, host

272 availability is often patchy and limited: fluctuations in environmental conditions and the

273 requirement to physically re-locate to find suitable host eggs pose barriers to constant

274 oviposition. Through manipulation of Trichogramma oviposition rates, by limiting access to host

275 eggs, we saw that patterns of offspring production had a significant effect on total sex ratio.

276 When wasps were not able to parasitize host eggs continuously, either by alternating days with

277 access to eggs, or limiting the time per day with egg access, sex ratios were maintained at higher

278 levels (Figure 2A). In fact, for wasps that had access to host eggs for only one hour a day, the

279 near-complete parthenogenesis phenotype was maintained for the duration of the trial, without

280 significant impact on total fecundity (Figure 2D). Critically, it is only in the first 24 hours where

281 treatment one wasps (constant host eggs) show drastically different fecundity than the treatment

282 three wasps (host eggs for one hour a day). On day two, mothers of these two treatments

283 produced nearly the same number of offspring, and for the remainder of the trial the treatment

284 three wasps produced higher numbers of offspring (Figure 2C). High fecundity within the first

28524 hours had a lasting effect on the sex ratio of progeny produced for the remainder of the trial. 
287 We show that it is not cumulative fecundity alone that determines the likelihood of the next

288 offspring being feminized (Figure 3). This corroborates the finding that there is no significant

289 difference in total fecundity between treatments. We see that sex ratios drop precipitously in

290 treatment one when approximately 45 offspring had been produced, significantly diverging from

291 the host-limited treatments. Even restricting access to hosts on only the first day has a prolonged

292 effect on the sex ratio of the offspring (Figure 5A).

293

294 Results from qPCR analysis of Wolbachia titers were mixed. It is worth noting that whole-body

295 extractions, which are necessary for the minute Trichogramma, likely do not provide the most

296 resolved look at Wolbachia titers in the ovaries, which could be responsible for symbiont

297 provisioning to the egg (Ferree et al. 2005). Regardless, Wolbachia titers were highest in

298 immediately collected wasps, which is congruent with our expectation (Figure 4A). The most

299 restrictive egg card access treatment maintained Wolbachia titers at a level comparable to those

300 of wasps who had yet to reproduce, indicating that Wolbachia titers had been sustained (Figure

301 4A). However, treatment two, which produced intermediate sex ratios, resulted in Wolbachia

302 titers that were indistinguishable from treatment one wasps that oviposited constantly, albeit

303 significantly lower than the immediately collected and treatment three wasps (Figure 4A). We

304 predict that this is reflective of the fact that wasps from both of those treatments were able to

305 oviposit up until their collection; whereas mothers from treatment three had 23 hours of recovery

306 prior to collection, resulting in Wolbachia titers similar to those that had yet to oviposit.

307

308 Wasps that oviposit relatively constantly may be maintaining a rate of germ line stem cell

309 turnover such that Wolbachia cannot keep up, resulting in fewer Wolbachia deposited into eggs, 
310 and the subsequent production of males. This is consistent with the finding that a large number

311 of Wolbachia are transferred from nurse cells into the developing oocyte (Ferree et al. 2005;

312 Serbus et al. 2008). Alternatively, Wolbachia from somatic tissues could continually invade the

313 germ line to ensure a high proportion of infected eggs (Fast et al. 2011; Toomey et al. 2013).

314 Under these circumstances, constant oviposition may draw Wolbachia from the soma resulting in

315 lower whole-body titers. We propose that the recovery periods built into our host access

316 treatments are critical to maintaining Wolbachia titers high enough in developing eggs to ensure

317 effective parthenogenesis induction. This is also in line with previous studies that showed a

318 positive relationship between Wolbachia titers and sex ratios in parthenogenesis-inducing

319 Wolbachia (Pascal et al. 2004; Stouthamer \& Mak 2002; Zchori-Fein et al. 2000).

320

321 Additional support for the hypothesis that Wolbachia titer is essential for parthenogenesis

322 induction comes from finding of lower Wolbachia titers in males compared to their sisters

323 (Figure 4B). While it is possible that adult titers may not be reflective of the number of

324 Wolbachia deposited into the egg, arguably, our data point to titers being important for proper

325 parthenogenesis-induction, which is again consistent with previous findings on the effect of heat

326 and antibiotics (Pascal et al. 2004; Pintureau et al. 1999; Stouthamer et al. 1990a), and the

327 importance of maternal loading of Wolbachia into eggs (Ferree et al. 2005; Serbus et al. 2008).

328 There is a chance that some of the phenotypic males with higher Wolbachia titer could be of

329 female karyotype, which has been shown to occur in related Trichogramma species and other

330 parthenogenesis-inducing Wolbachia infected wasps (Ma et al. 2015; Tulgetske 2010). We

331 would expect these individuals to have high enough Wolbachia titers to induce gamete 
332 duplication, but not high enough to result in the hypothesized epigenetic feminization that occurs

333 afterward (Tulgetske 2010).

334

335 It is likely that Wolbachia titers in the egg may not be the final determinant of successful

336 parthenogenesis induction, but instead it is a Wolbachia-secreted factor that needs to be at

337 sufficient levels. This has been hypothesized as a mechanism for the sex-ratio changes in

338 Muscidifurax (Zchori-Fein et al. 2000), and is the mechanism for inducing cytoplasmic

339 incompatibility, as sperm do not contain Wolbachia cells, but have been modified by Wolbachia-

340 derived proteins (Beckmann et al. 2017; LePage et al. 2017). Females from other closely related

341 species of Trichogramma hatch with a set of fully developed eggs, but will mature new eggs

342 over the course of their adult life (Volkoff \& Daumal 1994). The newly matured eggs may need

343 a longer "incubation time" in order to accumulate the appropriate concentration of Wolbachia or

344 a Wolbachia-derived parthenogenesis factor. More resolved studies of Wolbachia densities,

345 Wolbachia-protein densities, and the time that eggs spend in the mother, would aid in identifying

346 a threshold level of infection critical for effective parthenogenesis induction.

347

348 There is evidence for gene flow between populations of Trichogramma in the field, and that

349 Wolbachia-infected females can mate with males and fertilize their eggs (Stouthamer \& Kazmer

350 1994). Given that access to host egg resources has an impact on the likelihood of males being

351 produced, the amount of gene flow may fluctuate with environmental conditions. While limited

352 availability of host eggs is likely the norm, lepidopteran populations fluctuate, with abundance

353 peaking during certain seasons or in response to particular weather patterns (Kunte 1997; Pollard

354 1988; Roy et al. 2001; van den Bosch 2003). Environmental conditions could have direct effects 
355 on Wolbachia titers (such as high temperatures decreasing bacterial titers (Pintureau et al. 2002;

356 Stouthamer et al. 1990a)), and indirect effects through availability of host eggs. Greater host

357 availability may lead to an increase in offspring production, and if high enough, a decrease in sex

358 ratio. Males produced under these circumstances would likely provide a mechanism for gene

359 flow between asexual lineages.

360

361 The higher penetrance of parthenogenesis induction under host-limited conditions, as occurred in

362 our study can in part explain the frequent coexistence of infected and uninfected females in

363 Trichogramma field populations (Huigens et al. 2004; Stouthamer 1997; Stouthamer et al.

364 1990a). How these populations can coexist has been unclear, because laboratory experiments

365 with infected and uninfected lines from these field populations often showed that under constant

366 host availability (such as in our treatment one), the daughter production of infected females was

367 lower than that of mated uninfected females (Silva et al. 2000; Stouthamer \& Luck 1993).

368

369 In conclusion, we provide evidence for Trichogramma reproductive patterns mediating the

370 parthenogenesis phenotype, likely through the depletion of Wolbachia titers. The males produced

371 during times of high oviposition rates may provide an opportunity for gene flow between

372 populations, and thus new host-symbiont combinations. Given the interest in using Wolbachia as

373 a tool to control insect populations (Hoffmann et al. 2015; Turelli \& Hoffmann 1991), it is

374 critical that we understand the context-dependent nature of Wolbachia phenotypes, and how this

375 may result in different selective pressures for the host-symbiont relationship.

376

377 


\section{Acknowledgments}

379 We thank Barbara Baker and Christina Luu for their assistance in collecting many tiny wasps

380 into ethanol, and Sarah Lillian for her statistical advice. We also thank Paul Rugman-Jones, Eric

381 Smith, Matthew Daugherty, Jason Stajich, and three reviewers for helpful discussions and

382 feedback on drafts of the manuscript.

383 


\section{References}

385 Aron S, de Menten L, Van Bockstaele DR, Blank SM, and Roisin Y. 2005. When hymenopteran males reinvented diploidy. Current Biology 15:824-827. 10.1016/j.cub.2005.03.017

387 388 Eigen and S4. R package version 1.

Bordenstein SR, and Bordenstein SR. 2011. Temperature affects the tripartite interactions between bacteriophage WO, Wolbachia, and cytoplasmic incompatibility. PLoS One 6:e29106.

Bordenstein SR, Uy JJ, and Werren JH. 2003. Host genotype determines cytoplasmic incompatibility type in the haplodiploid genus Nasonia. Genetics 164:223-233.

Bourtzis K, Dobson SL, Xi Z, Rasgon JL, Calvitti M, Moreira LA, Bossin HC, Moretti R, Baton LA, Hughes GL, Mavingui P, and Gilles JRL. 2014. Harnessing mosquito-Wolbachia symbiosis for vector and disease control. Acta Tropica 132, Supplement:S150-S163. http://dx.doi.org/10.1016/j.actatropica.2013.11.004

Bourtzis K, Nirgianaki A, Markakis G, and Savakis C. 1996. Wolbachia infection and cytoplasmic incompatibility in Drosophila species. Genetics 144:1063-1073.

Breeuwer J, and Werren JH. 1993. Cytoplasmic incompatibility and bacterial density in Nasonia vitripennis. Genetics 135:565-574.

Clancy DJ, and Hoffmann AA. 1998. Environmental effects on cytoplasmic incompatibility and bacterial load in Wolbachia - infected Drosophila simulans. Entomologia Experimentalis et Applicata 86:13-24. 
405 Fast EM, Toomey ME, Panaram K, Desjardins D, Kolaczyk ED, and Frydman HM. 2011.

406 Wolbachia enhance Drosophila stem cell proliferation and target the germline stem cell $407 \quad$ niche. Science 334:990-992.

408 Ferree PM, Frydman HM, Li JM, Cao J, Wieschaus E, and Sullivan W. 2005. Wolbachia utilizes 409 host microtubules and Dynein for anterior localization in the Drosophila oocyte. PLoS

410 Pathogens 1:e14.

411 Gottlieb Y, Zchori-Fein E, Werren JH, and Karr TL. 2002. Diploidy restoration in Wolbachia412 infected Muscidifurax uniraptor (Hymenoptera: Pteromalidae). Journal of Invertebrate

414 Grenier S, Bernard P, Heddi A, Lassabliére F, Jager C, Louis C, and Khatchadourian C. 1998. 415 Successful horizontal transfer of Wolbachia symbionts between Trichogramma wasps. Proceedings of the Royal Society B-Biological Sciences 265:1441-1445. 10.1098/rspb.1998.0455

Hoffmann AA, Montgomery BL, Popovici J, Iturbe-Ormaetxe I, Johnson PH, Muzzi F, Greenfield M, Durkan M, Leong YS, Dong Y, Cook H, Axford J, Callahan AG, Kenny N, Omodei C, McGraw EA, Ryan PA, Ritchie SA, Turelli M, and O'Neill SL. 2011. Successful establishment of Wolbachia in Aedes populations to suppress dengue transmission. Nature 476:454-U107. 10.1038/nature10356

Hoffmann AA, Ross PA, and Rasic G. 2015. Wolbachia strains for disease control: ecological 424 and evolutionary considerations. Ecology and Evolution 8:751-768. 10.1111/eva.12286

425 Hoffmann AA, Turelli M, and Harshman LG. 1990. Factors affecting the distribution of 426 cytoplasmic incompatibility in Drosophila simulans. Genetics 126:933-948. 
427 Hohmann CL, Luck RF, and Stouthamer R. 2001. Host deprivation effect on reproduction and

$428 \quad$ survival of Wolbachia-infected and uninfected Trichogramma kaykai Pinto \& Stouthamer

429 (Hymenoptera: Trichogrammatidae). Neotropical Entomology 30:601-605.

430 $10.1590 / \mathrm{S} 1519-566 \times 2001000400014$

431 Huigens ME, de Almeida RP, Boons PAH, Luck RF, and Stouthamer R. 2004. Natural

432

433

434

435

436

437

438

439

440

441

442

443

444

445

446

447

448

449 interspecific and intraspecific horizontal transfer of parthenogenesis-inducing Wolbachia in Trichogramma wasps. Proceedings of the Royal Society B-Biological Sciences 271:509-515. 10.1098/rspb.2003.2640

Hurst GD, Johnson AP, vd Schulenburg JHG, and Fuyama Y. 2000. Male-killing Wolbachia in Drosophila: a temperature-sensitive trait with a threshold bacterial density. Genetics 156:699-709.

Ikeda T, Ishikawa H, and Sasaki T. 2003. Infection density of Wolbachia and level of cytoplasmic incompatibility in the Mediterranean flour moth, Ephestia kuehniella. Journal of Invertebrate Pathology 84:1-5.

Kunte KJ. 1997. Seasonal patterns in butterfly abundance and species diversity in four tropical habitats in northern Western Ghats. Journal of biosciences 22:593-603. 10.1007/BF02703397

Legner E. 1985. Natural and induced sex ratio changes in populations of thelytokous Muscidifurax uniraptor (Hymenoptera: Pteromalidae). Annals of the Entomological Society of America 78:398-402. 10.1093/aesa/78.3.398

Lindsey ARI, Werren JH, Richards S, and Stouthamer R. 2016. Comparative genomics of a parthenogenesis-inducing Wolbachia symbiont. G3: Genes $\mid$ Genomes $\mid$ Genetics. $10.1534 / \mathrm{g} 3.116 .028449$ 
450 Livak KJ, and Schmittgen TD. 2001. Analysis of relative gene expression data using real-time

451

452

453

454

455

456

457

458

459

460

461

462

463

464

465

466

467

468

469

470 quantitative PCR and the 2- $\Delta \Delta \mathrm{CT}$ method. methods 25:402-408. 10.1006/meth.2001.1262

Ma WJ, Pannebakker BA, van de Zande L, Schwander T, Wertheim B, and Beukeboom LW. 2015. Diploid males support a two-step mechanism of endosymbiont-induced thelytoky in a parasitoid wasp. BMC Evolutionary Biology 15:84. 10.1186/s12862-015-0370-9

McGraw E, Merritt D, Droller J, and O'Neill S. 2001. Wolbachia-mediated sperm modification is dependent on the host genotype in Drosophila. Proceedings of the Royal Society of London B: Biological Sciences 268:2565-2570.

Mouton L, Henri H, Charif D, Boulétreau M, and Vavre F. 2007. Interaction between host genotype and environmental conditions affects bacterial density in Wolbachia symbiosis. Biology Letters 3:210-213.

Newton IL, Savytskyy O, and Sheehan KB. 2015. Wolbachia utilize host actin for efficient maternal transmission in Drosophila melanogaster. PLoS Pathogens 11:e1004798. 10.1371/journal.ppat. 1004798

Pannebakker BA, Pijnacker LP, Zwaan BJ, and Beukeboom LW. 2004. Cytology of Wolbachiainduced parthenogenesis in Leptopilina clavipes (Hymenoptera: Figitidae). Genome 47:299-303. 10.1139/g03-137

Pascal C, Pintureau B, Charles H, Katchadourian C, Grenier S, Bolland P, and Robin C. 2004. Relationship between Wolbachia density and sex-ratio in a Trichogramma strain. Agrociencia 8:11-22. 
471 Pintureau B, Chapelle L, and Delobel B. 1999. Effects of repeated thermic and antibiotic

472 treatments on a Trichogramma (Hym., Trichogrammatidae) symbiont. Journal of Applied

473 Entomology 123:473-483.

474 Pintureau B, Lassabliere F, Daumal J, and Grenier S. 2002. Does a cyclic natural thermal cure 475 occur in Wolbachia - infected Trichogramma species? Ecological Entomology 27:366-

476 372.

477

478

479

480

481

482

483

484

485

486

487

488

489

490

491

492

493 828. $10.2307 / 2403748$

Reynolds KT, Thomson LJ, and Hoffmann AA. 2003. The effects of host age, host nuclear background and temperature on phenotypic effects of the virulent Wolbachia strain popcorn in Drosophila melanogaster. Genetics 164:1027-1034.

Roy DB, Rothery P, Moss D, Pollard E, and Thomas J. 2001. Butterfly numbers and weather: predicting historical trends in abundance and the future effects of climate change. Journal of Animal Ecology 70:201-217. 10.1111/j.1365-2656.2001.00480.x

Serbus LR, Casper-Lindley C, Landmann F, and Sullivan W. 2008. The genetics and cell biology of Wolbachia-host interactions. Annual Review of Genetics 42:683-707.

\subsection{6/annurev.genet.41.110306.130354}

Serbus LR, White PM, Silva JP, Rabe A, Teixeira L, Albertson R, and Sullivan W. 2015. The impact of host diet on Wolbachia titer in Drosophila. PLoS Pathog 11:e1004777.

Silva I, Van Meer MMM, Roskam MM, Hoogenboom A, Gort G, and Stouthamer R. 2000. Biological control potential of Wolbachia-infected versus uninfected wasps: Laboratory and greenhouse evaluation of Trichogramma cordubensis and T. deion strains. Biocontrol Science and Technology 10:223-238. 10.1080/09583150050044501 
494 Stouthamer R. 1997. Wolbachia-induced parthenogenesis.

495 Stouthamer R, Breeuwer JAJ, Luck RF, and Werren JH. 1993. Molecular-identification of 496 microorganisms associated with parthenogenesis. Nature 361:66-68. 10.1038/361066a0

497 Stouthamer R, Hu JG, van Kan F, Platner GR, and Pinto JD. 1999. The utility of internally 498 transcribed spacer 2 DNA sequences of the nuclear ribosomal gene for distinguishing 499 sibling species of Trichogramma. BioControl 43:421-440. 10.1023/a:1009937108715

500 Stouthamer R, and Kazmer DJ. 1994. Cytogenetics of microbe-associated parthenogenesis and 501

502 its consequences for gene flow in Trichogramma wasps. Heredity 73:317-327. 10.1038/hdy.1994.139

503 Stouthamer R, and Luck R. 1993. Influence of microbe-associated parthenogenesis on the 504 fecundity of Trichogramma deion and T. pretiosum. Entomologia Experimentalis et 505 Applicata 67:183-192. 10.1111/j.1570-7458.1993.tb01667.x

506 Stouthamer R, Luck RF, and Hamilton WD. 1990a. Antibiotics cause parthenogenetic

507 Trichogramma (Hymenoptera, Trichogrammatidae) to revert to sex. Proceedings of the 508 National Academy of Sciences 87:2424-2427. 10.1073/pnas.87.7.2424

509

510

511

512

513

514

515 516

Stouthamer R, and Mak F. 2002. Influence of antibiotics on the offspring production of the Wolbachia-infected parthenogenetic parasitoid Encarsia formosa. Journal of Invertebrate Pathology 80:41-45.

Stouthamer R, Pinto JD, Platner GR, and Luck RF. 1990b. Taxonomic status of thelytokous forms of Trichogramma (Hymenoptera: Trichogrammatidae). Annals of the Entomological Society of America 83:475-481. 10.1093/aesa/83.3.475

Toomey ME, Panaram K, Fast EM, Beatty C, and Frydman HM. 2013. Evolutionarily conserved Wolbachia-encoded factors control pattern of stem-cell niche tropism in Drosophila 
ovaries and favor infection. Proceedings of the National Academy of Sciences 110:10788-

518 10793.

519 Tulgetske GM. 2010. Investigations into the mechanisms of Wolbachia induced parthenogenesis 520 and sex determination in the parasitoid wasp, Trichogramma: Ph.D. Dissertation, $521 \quad$ University of California, Riverside.

522 Tulgetske GM, and Stouthamer R. 2012. Characterization of intersex production in 523 Trichogramma kaykai infected with parthenogenesis-inducing Wolbachia. Naturwissenschaften 99:143-152. 10.1007/s00114-011-0880-2

525 Turelli M, and Hoffmann AA. 1991. Rapid spread of an inherited incompatibility factor in 526 California Drosophila. Nature 353:440-442.

527 Turelli M, and Hoffmann AA. 1995. Cytoplasmic incompatibility in Drosophila simulans: 528 dynamics and parameter estimates from natural populations. Genetics 140:1319-1338.

529 Untergasser A, Cutcutache I, Koressaar T, Ye J, Faircloth BC, Remm M, and Rozen SG. 2012.

$530 \quad$ Primer3 - new capabilities and interfaces. Nucleic Acids Research 40:e115-e115. 10.1093/nar/gks596

532

van den Bosch R. 2003. Fluctuations of Vanessa cardui butterfly abundance with El Nino and Pacific Decadal Oscillation climatic variables. Global Change Biology 9:785-790.

$$
\text { 10.1046/j.1365-2486.2003.00621.x }
$$

Volkoff A, and Daumal J. 1994. Ovarian cycle in immature and adult stages of Trichogramma cacoeciae and T. brassicae (Hym.: Trichogrammatidae). BioControl 39:303-312. 10.1007/BF02373035

Walker T, Johnson PH, Moreira LA, Iturbe-Ormaetxe I, Frentiu FD, McMeniman CJ, Leong YS, Dong Y, Axford J, Kriesner P, Lloyd AL, Ritchie SA, O'Neill SL, and Hoffmann AA. 
2011. The $w$ Mel Wolbachia strain blocks dengue and invades caged Aedes aegypti populations. Nature 476:450-U101. 10.1038/nature10355

542 Walsh PS, Metzger DA, and Higuchi R. 1991. Chelex 100 as a medium for simple extraction of DNA for PCR-based typing from forensic material. BioTechniques 10:506-513.

544 Watanabe M, Kageyama D, and Miura K. 2013. Transfer of a parthenogenesis-inducing

545

546

547

548

549

550

551

552

553

554

555

556

557

558

559

560

561

562

Wolbachia endosymbiont derived from Trichogramma dendrolimi into Trichogramma evanescens. Journal of Invertebrate Pathology 112:83-87. http://dx.doi.org/10.1016/j.jip.2012.09.006

Werren JH, Baldo L, and Clark ME. 2008. Wolbachia: master manipulators of invertebrate biology. Nature Reviews Microbiology 6:741-751. 10.1038/nrmicro1969

Werren JH, and Windsor DM. 2000. Wolbachia infection frequencies in insects: Evidence of a global equilibrium? Proceedings of the Royal Society B-Biological Sciences 267:12771285. $10.1098 /$ rspb.2000.1139

Wiwatanaratanabutr I, and Grandjean F. 2016. Impacts of temperature and crowding on sex ratio, fecundity and Wolbachia infection intensity in the copepod, Mesocyclops thermocyclopoides. Journal of Invertebrate Pathology 141:18-23.

Ye J, Coulouris G, Zaretskaya I, Cutcutache I, Rozen S, and Madden TL. 2012. Primer-BLAST: a tool to design target-specific primers for polymerase chain reaction. $B M C$ Bioinformatics 13:1. 10.1186/1471-2105-13-134

Zchori-Fein E, Gottlieb Y, and Coll M. 2000. Wolbachia density and host fitness components in Muscidifurax uniraptor (Hymenoptera: Pteromalidae). Journal of Invertebrate Pathology 75:267-272. 10.1006/jipa.2000.4927 
Figure 1 (on next page)

Experimental design for host access treatments.

A) Treatment One: a fresh egg card every 24 hours; wasps have constant access to host eggs. Treatment Two: one day on, one day off; wasps have constant access to host eggs every other day. Treatment Three: wasps have access to a fresh egg card for only one hour a day. Treatment Four: collect adult wasps into ethanol immediately upon emergence. B) Control: a fresh egg card every 24 hours; wasps have constant access to host eggs (same as treatment one in 1A). Experimental: wasps have access to a fresh egg card for only one hour on day one, followed by constant access to host eggs starting day two, with a egg cards exchanged every 24 hours. 


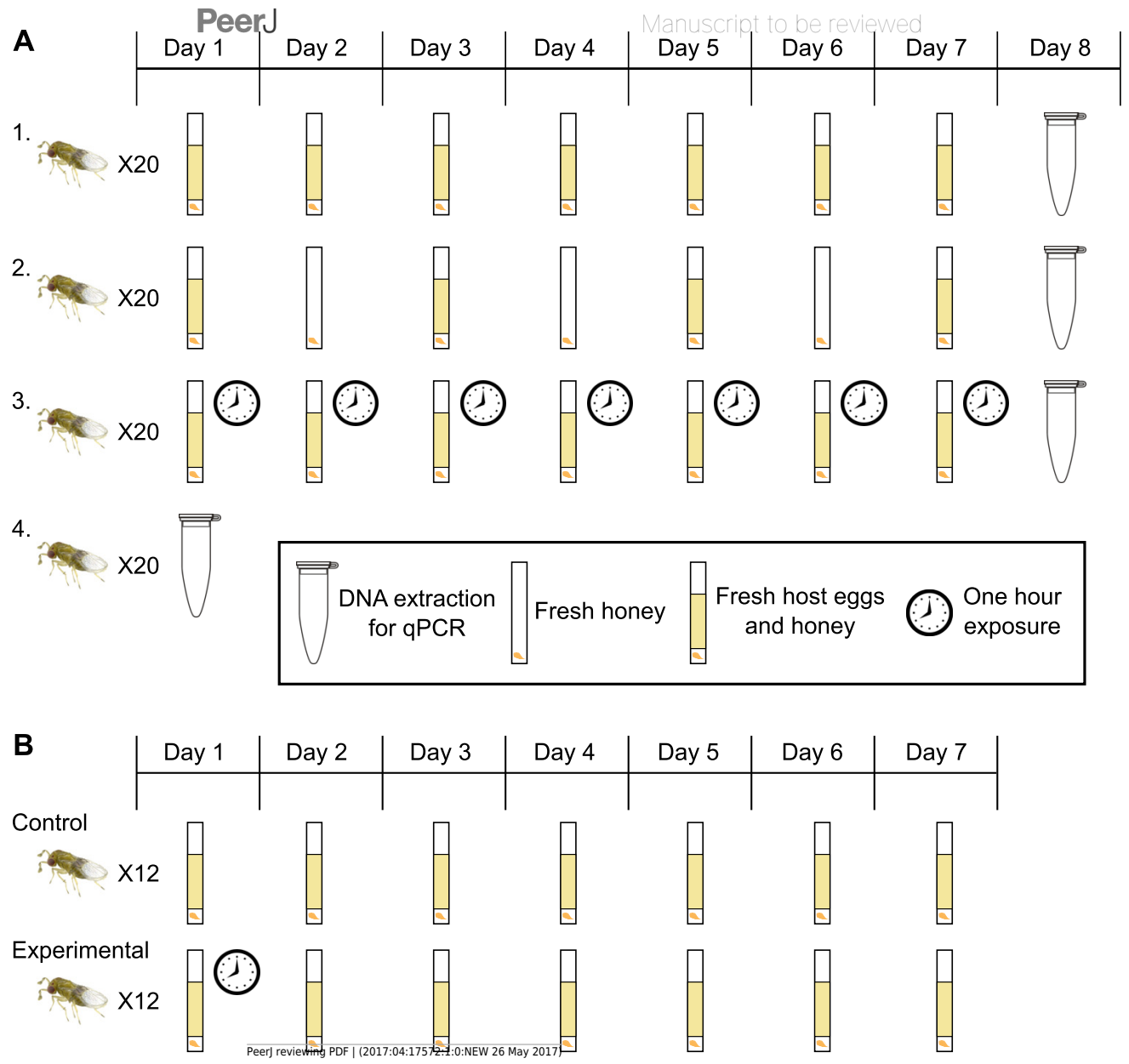




\section{Figure 2 (on next page)}

Sex ratios and fecundity for host access treatments shown in Figure 1A.

In panels $B$ and $D$, open circles represent outliers, double asterisks represent $p \leq 0.01$, and triple asterisks represent $p \leq 0.001$. A) Temporal variation in sex ratio. B) Total sex ratio for the seven-day period. C) Temporal variation in fecundity. D) Total fecundity for the seven-day period. 


\section{Figure 3 (on next page)}

Cumulative fecundity and sex ratios for host access treatments.

The left-most point of each line represents the first day of the trial, and the right-most point represents the last day of the trial. 


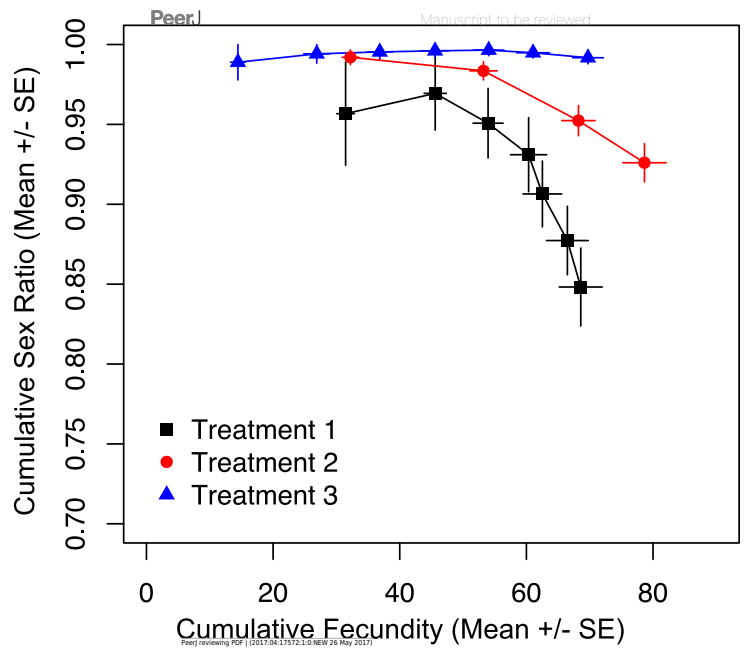




\section{Figure 4(on next page)}

Wolbachia titers, as measured by quantification of $16 \mathrm{~S}$ relative to wingless.

Within a plot, titers have been normalized to the sample shown most left. Open circles represent outliers, a single asterisk represents $p \leq 0.05$ and double asterisks represent $p \leq$ 0.01. A) Wolbachia titers of mothers collected after the host access treatments one through four. Only significant pairwise comparisons are denoted. B) Wolbachia titers of the offspring produced by mothers subjected to treatment one. Point styles denote offspring that originated from the same mother. 


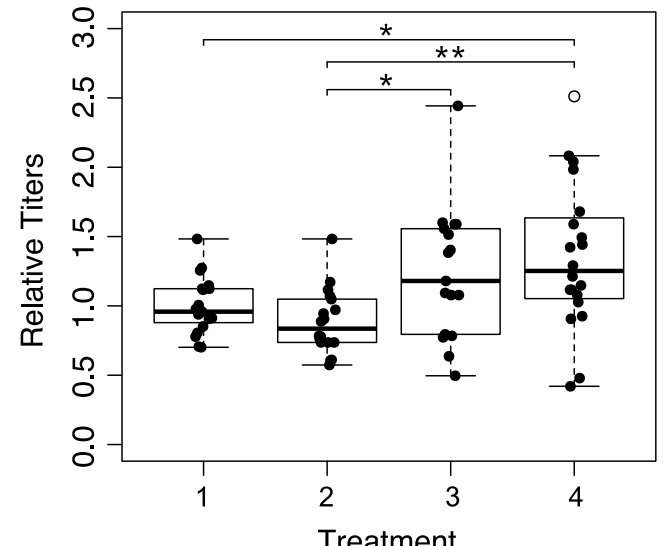

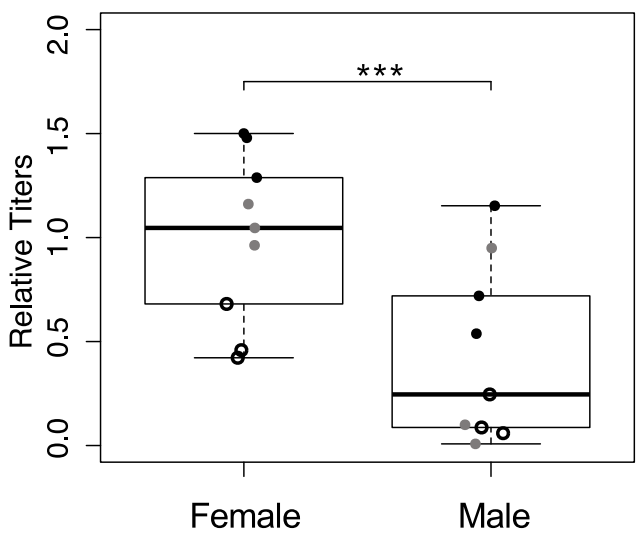

B Manuscript to be reviewed 


\section{Figure 5 (on next page)}

Sex ratios and fecundity for host access treatments shown in Figure 1B.

In panels $B$ and $D$, open circles represent outliers and triple asterisks represent $p \leq 0.001$. A) Temporal variation in sex ratio. B) Total sex ratio for the seven-day period. C) Temporal variation in fecundity. D) Total fecundity for the seven-day period. 


\section{$\mathbf{A}$}

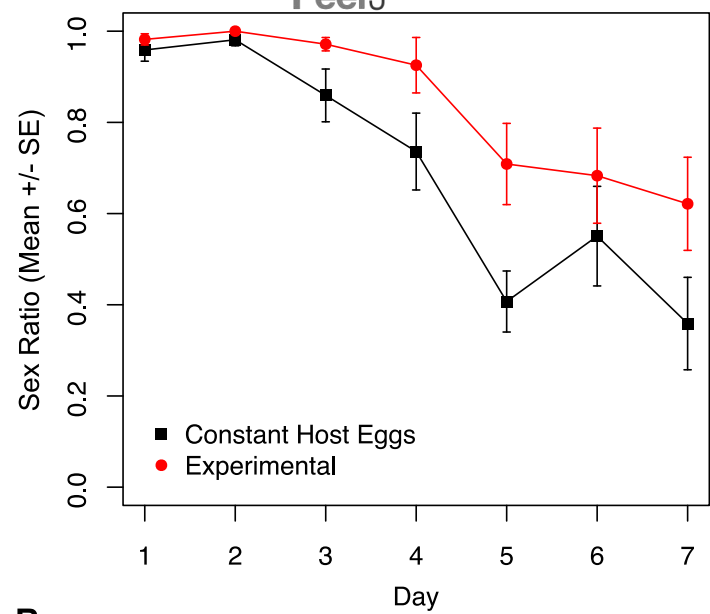

B

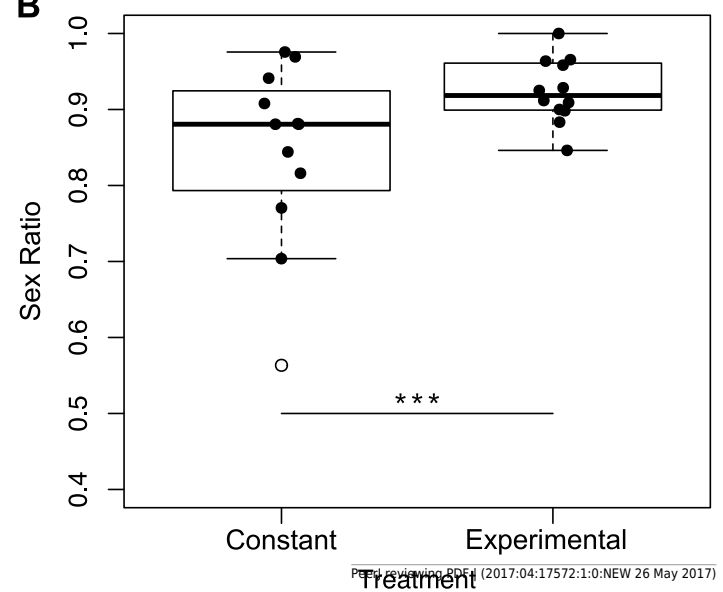

C Manuscript to be reviewed

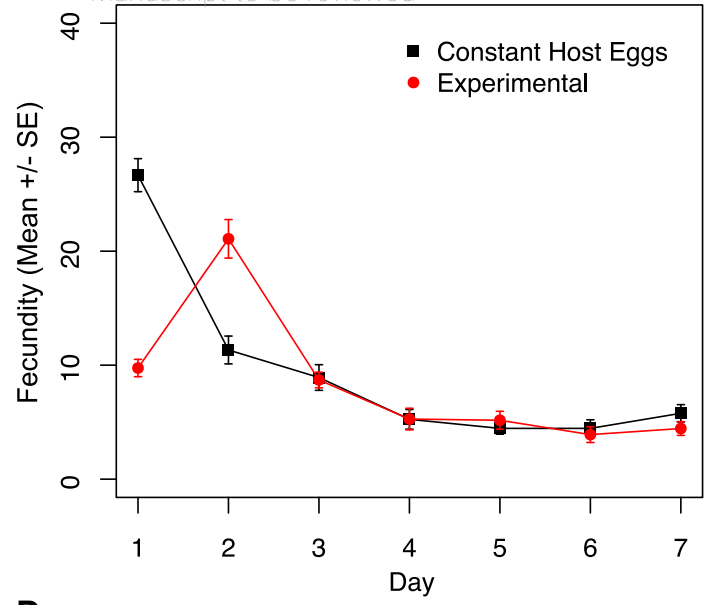

D

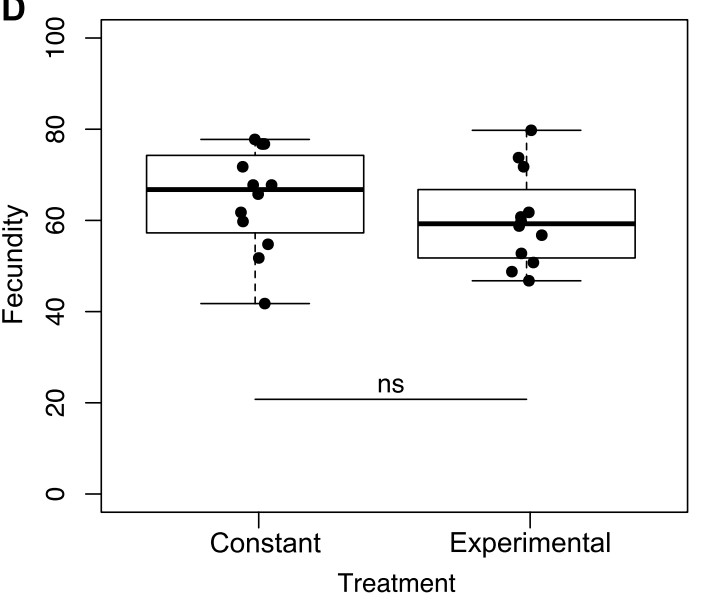




\section{Table $\mathbf{1}$ (on next page)}

Sequences of primers used in this study. 
1 Table 1. Sequences of primers used in this study.

\begin{tabular}{cccc}
\hline Locus & Primer & Sequence (5' to 3') & Amplicon Size \\
\hline \multirow{2}{*}{$16 \mathrm{~S}$} & $16 \mathrm{~S} \_\mathrm{qF}$ & GAG GAA GGT GGG GAT GAT GTC & $103 \mathrm{bp}$ \\
& $16 \mathrm{~S} \_\mathrm{qR}$ & CTT AGG CTT GCG CAC CTT G & \\
\multirow{2}{*}{ wingless } & wg_qF & AGC TCA AGC CCT ACA ATC CG & 99bp \\
& wg_qR & CCA GCT TGG GGT TCT TCT CG & \\
\hline
\end{tabular}

2 